\title{
Space, Linguistic Expression of
}

Penelope Brown, Max Planck Institute for Psycholinguistics, Nijmegen, The Netherlands

(c) 2015 Elsevier Ltd. All rights reserved.

This article is a revision of the previous edition article by S.C. Levinson, volume 22, pp. 14749-14752, (c) 2001, Elsevier Ltd.

\begin{abstract}
Spatial cognition is central to human thinking, and spatial language is thus an important area of study, as it may reveal fundamental properties of human thought. Recent research has shown that spatial language is much more divergent across languages than had previously been thought, suggesting significant cultural patterning of spatial conceptualization. This article reviews spatial language cross-linguistically, sets out a typological framework for the language of space, and considers the relationship of spatial language to spatial cognition, in the context of extensive linguistic diversity in the spatial domain
\end{abstract}

Spatial cognition is at the heart of much human thinking, as evidenced, for example, in the explanatory power of spatial metaphors and diagrams, and the explication of spatial concepts has played a key role in the development of Western mathematics and philosophy over two and a half millennia (see Jammer, 1954). It is therefore easy to assume that spatial notions form a robust, universal core of human cognition; this assumption has dominated the cognitive sciences (e.g., Miller and Johnson-Laird, 1976). But naive human spatial language turns out to vary significantly across languages, both in the way it is semantically organized and the way in which it is coded. This has major implications for how we should think about universals in human thought and language.

The Western intellectual tradition has provided an elaborate metalanguage for spatial concepts, but the conceptual underpinnings of naive human spatial language utilize only a small subset of these concepts. Moreover, there are many aspects of our spatial perception and motor control which utilize much richer and more precise representations of space than are coded in language. The study of the representation of space in language, and its relation to cognition, must therefore be treated as an empirical enterprise, and this article reports on the increasing body of cross-linguistic information about how different languages structure the spatial domain (see Levinson and Wilkins, 2006).

\section{Space as a Semantic Domain}

Languages tend not to treat space as a single, coherent semantic field in the same way that they treat, for example, color (q.v.), kinship (q.v.), or ethnobotany - rather, space is structured as a large semantic field with many distinct subdomains, each of which is closely structured (and often varies considerably across languages). Nearly all languages have 'where' questions that cover the entire field (i.e., with the same or morphologically related forms for 'where-to,' 'where-from,' and 'where-at') and describe the location of one object primarily with respect to another. But beyond these general properties, spatial expressions are structured in distinct subdomains, notably topology, frames of reference, and motion, with place names and spatial deixis as complementary areas. These subdomains cross-cut in certain ways (e.g., frames of reference may play a role in motion description), but because they have their own internal organization they need to be kept analytically distinct.

Although the philosophical and scientific literature gives us many different ways to talk about space, natural languages tend to structure spatial description more in accord with the ideas of Leibniz than of Newton. That is, location is thought about as the place of one thing relative to another (rather than, as in Newtonian thinking, as specifications in the coordinates of an infinite, abstract three-dimensional 'box'). We will call the object to be located the 'figure' (F), and the object with respect to which the location is specified the 'ground' $(G)$; other equivalent terms in the literature are 'theme' versus 'relatum' or 'trajector' versus 'landmark.' As Talmy (1983) noted, a good ground or landmark object is larger than and less mobile than the figure object to be located. The problems of location specification then largely boil down to the following functional considerations:

1. Where figure $F$ and ground $G$ are contiguous or coincident, a static F may be said to be 'at G.' Where G is large, it can be subdivided, so that $\mathrm{F}$ can be said to be 'at the X-part of G.' This is, roughly, the subdomain of topology.

2. Where $F$ and $G$ are separated in space, the problem is to specify an angle or direction from $G$ in which $F$ can be found (the search domain). This involves a coordinate system of some kind - a 'frame of reference' - of which there are three main types found in languages.

3. Where F is in motion, two kinds of ground are especially relevant: the source and the goal of the motion. To indicate the progression of motion (i.e., the increase or decrease of distance from a ground), motion can be specified as ' $\mathrm{F}$ is moving toward goal $\mathrm{G}^{\prime}$ or 'F is moving from source S.' In addition, motion can be thought of as bringing about, or destroying, a topological relation, as in 'F moves into/out of G.' But to indicate a direction or vector of motion, both $\mathrm{S}$ and $\mathrm{G}$ must be specified, or a coordinate system of the kind mentioned in (2) utilized. Of course, motion can also be specified as taking place within a location, in which case the systems in (1) and (2) can be utilized.

These functional distinctions give us a number of cleavages: stasis (or location) versus kinesis (or motion), nonangular topological relationships versus angular relations specified in 


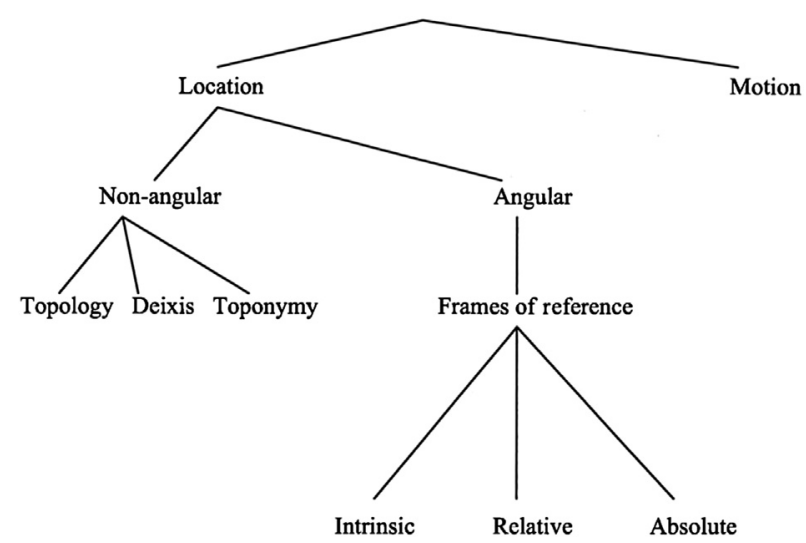

Figure 1 Crosscutting subdomains of spatial language.

frames of reference, with potentially cross-cutting domains as shown in Figure 1.

Some further details in Figure 1, like the three frames of reference, are described below. But the two subfields that have not been discussed further need to be mentioned here. One is deixis, which concerns the way in which the location of the speech participants can constitute a special ground or landmark (as in here), which then makes interpretation dependent on determining that location (as in He used to come here). Languages universally seem to provide a structured set of deictic elements with distinctions that are often spatial (demonstratives like 'this' vs 'that,' adverbs like 'here' vs 'there'). Deictic anchoring of spatial expressions is, however, often implicit or pragmatic: the local pub implies a pub close to some reference point, which unless specified, is taken to be the place of speaking.

A second subfield not treated further here is toponymy, or systems of place names. Place names have been much investigated from an historical point of view; since they are highly conservative they reveal a great deal about the locations of ancient languages and peoples. However, the theory and crosslinguistic typology of place names - e.g., why some are descriptive, others not - is hardly developed at all (see e.g., Hunn, 1993).

\section{Universals and Variation in the Semantic Parameters in Spatial Subdomains}

In this section, the semantic parameters involved in spatial description in two major subdomains are described to illustrate some of the different ways in which languages structure the spatial domain.

\section{Topology}

The subdomain of topology, as mentioned, concerns contiguous relations between figure and ground. The term is potentially misleading - topology proper is a branch of geometry concerned with constancies under continuous distortions such as stretching, but, following Piaget (Piaget and Inhelder, 1956), it is used in studies of spatial language to describe the sort of spatial relations covered by the English prepositions on, in, and at (Herskovits, 1986). These spatial terms are among the earliest learnt by Western children, and it has therefore been supposed that they form a universal innate core of spatial notions. However, cross-linguistic studies show that universal semantic concepts are only to be found at a more abstract level. Take, for example, the spatial relations covered by English on, as in The cup is on the table, The picture is on the wall, or The fresco is on the ceiling - clearly the term on covers a wide area which is already subdivided in the adpositions (prepositions or postpositions) of many other European languages. Some languages (like Japanese) conflate 'on' relations with 'over' relations (as in The light is over the table) in their adpositions; others (like Yukatek Maya) even include 'under' relations as well (as in The ball is under the table). A detailed analysis of a dozen languages from different families suggests that universal semantic concepts in this domain lie at a more atomic level (Levinson and Wilkins, 2006). If we think of the core prototype of English on as consisting of the notion of vertical superposition of figure over ground, with a horizontal ground supporting a nonattached figure, then the kind of notions that look universal are at the level of 'vertical superposition,' 'contact,' 'horizontal supporting surface,' and (non)'attachment.' Clearly, these more abstract atomic concepts can be rearranged in many different ways to constitute the semantic relations expressible in an individual language (Levinson and Meira, 2003).

Topological relations are coded in the European languages primarily in adpositions or case, but, as we shall see, these are not the only possibilities - some languages code topological relations entirely in nouns or verbs.

\section{Frames of Reference}

Linguists have presumed that there are two main frames of reference, which are often called 'deictic' and 'intrinsic,' and which can be illustrated by the ambiguity of the sentence The boy is behind the bus - either the bus is between the boy and us ('deictic') or he is at the rear of the bus ('intrinsic'). Psychologists have long known, however, that on the vertical dimension our perceptual systems use three frames of reference against which to measure uprightness: an object-centered frame, a viewercentered frame, and an environmental frame. Cross-linguistic research shows that in fact these three frames of reference, which we will call the intrinsic, the relative, and the absolute frame, respectively, may be used on the horizontal plane too (Levinson, 1996). These three frames appear to be universally available, yet not all languages make systematic use of them all some use the absolute frame, others the relative frame, while most use the intrinsic frame, and some use all three. The main universal constraint appears to be that the relative frame is dependent on the possession of the intrinsic frame.

The intrinsic frame of reference, as its name implies, has to do with the intrinsic parts of ground objects. Recollect that frames of reference are coordinate systems that specify angles or directions from a landmark. One way to achieve this is to designate a side or facet of an object, so that the figure can be said to be located on an axis drawn from the center of the object through the designated side. Most languages provide a vocabulary for parts of objects, but the ways in which they partition objects can be very variable. In English, for example, the criteria 
for assigning the front of an object involve a complex mix of functional and orientational information: the front of a bus is the direction in which it canonically moves, the front of a building is the side in which it is normally entered, and the front of a television the side designed to be watched. In English the top of a bottle remains the top even when tipped over, but in other languages (like Zapotec) a more strictly orientational approach may assign the 'top' to whatever side is currently uppermost. Alternatively (as in Tzeltal), the assignment may rely entirely on the internal axial geometry of the object, so that, for example, the 'face' of an object may be a flat side on a secondary axis, even if normally this is undermost. What all these systems have in common is that they provide a system of designated sides to objects which is independent of the position of the viewer, and (apart from the vertical axis) independent of an environmental orientation. How they achieve this differs not only in detail but also in fundamentals.

Another way of obtaining angular distinctions on the horizontal plane is to use the body axes of the viewer. We can then say that something is, for example, to the 'left' of something else in the visual field. Full versions of such systems use a left/right axis and an orthogonal front/back axis, but again the way in which they map bodily axes onto the ground object is very variable. Although these systems are often called 'deictic' in the literature, this is misleading - they typically have a deictic center but they do not have to (as in He kicked the ball to the left of the goal), and the other two frames of reference can also have deictic centers (e.g., It is north of us,' 'It is at my left hand'). Relative frames of reference are, here, said to be 'relative' because they are relative to the body axes of some observer. Some languages translate the bodily coordinates onto the ground object without rotation; in such languages (like Hausa) The boy is to the left of the tree means just what it does in English, but The boy is in front of the tree means what in English is described as The boy is behind the tree. In contrast, some languages rotate the axes, so that the front of the tree is toward us, but the left of the tree is what in English we would call its right (this is probably a minority option, but is found in some dialects of Tamil). Some languages reflect the axes, as in English, so that left and right remain in the same directions as the viewer's body, but front and back are reversed or 'flipped' over.

Notice that terms like 'front' and 'back' and 'left' and 'right' also have intrinsic interpretations (as in He's sitting at the chairman's left), and for that reason ambiguities arise of the kind illustrated above with The boy is behind the bus. Relative systems are based on the intrinsic sides of humans, and probably arise as extensions of an intrinsic system to objects (like trees or balls) which resist the assignment of named sides. Hence the implicational relation: all relative systems are associated with intrinsic systems, but the possession of an intrinsic system does not necessarily imply the use of a relative one. Relative systems had been thought to be universal, but in fact it turns out that perhaps as many as a third of the world's languages do not employ them, instead making use of absolute systems.

Absolute systems rely on environmental gradients or 'geocentric' coordinates. Such systems use absolute directions, or fixed bearings, a bit like English north, but unlike in English, they are often used on all scales (as in The cup to the north of yours is mine, or even There's a spider on your northern leg), completely replacing the need for relative systems. There are many different varieties of absolute systems. Some of them (as in Australian
Aboriginal languages) are fully abstract cardinal direction systems, with terms that may gloss 'north,' 'south,' etc., but which denote quadrants or arcs of specific angle, and are often based on bearings rotated from our cardinal directions. To use a system of this kind, speakers must always orient themselves precisely, using multiple environmental clues (wind directions, solar angles, sidereal ecliptics, landscape features, and the like). Other systems are based more directly on one major environmental cue, like prevailing winds (as in languages of the Torres Straits), river drainage (as in Alaska), a seaward/landward distinction (as in many Oceanic languages), or prevailing slopes in mountainous terrain (as in the Himalayas). For example, Tenejapan Tzeltal uses an 'uphill,' 'downhill,' 'across' system, based on the general lay of the land in the mountainous territory of Tenejapa (Chiapas, Mexico), but the system has been abstracted from the terrain to provide a fixed orientation, so that the bearings remain constant outside the territory. Many systems use fixed orthogonal axes, but not all; for example, the Austronesian languages in the Pacific islands typically use the monsoon winds as the basis for one axis, but an inland-sea opposition as the basis for another axis; as one moves around the island, the angles between the axes constantly change.

It is important to note that there are cognitive consequences of relying on an absolute frame of reference for routine spatial description on all scales. Communication using such an absolute system requires that speakers' mental computations are adapted to enable constant background tracking of the coordinate system(s) and 'dead-reckoning,' or updating of speakers' locations as they move through the landscape (Levinson, 2003). Various cultural practices will support this background tracking (e.g., absolutely oriented gestures, attention to movements of the sun, shadows, and distant landmarks). Studies on the use of pointing gestures in cultures where absolute systems dominate in everyday use have contributed greatly to our understanding of how spatial frames of reference are invoked, communicated, and switched in conversational interaction, and provided important insights into the cognitive background to absolute systems (e.g., Levinson, 1997; Haviland, 2000, 2003; Kita et al., 2001; Le Guen, 2011). They have shown that gesture is deeply integrated into the system of directional reference, making it clear that these are not simply linguistic systems but broader communicative ones.

This raises the question of how children learn to use such apparently cognitively demanding systems. There have been a number of studies of children's acquisition of absolute systems (de León, 1994; Brown, 2001; Brown and Levinson, 2000, 2009; Cablitz, 2002; Dasen and Mishra, 2010). These have generally found that children master the basics of their absolute system relatively early - by the age of 6 or 7 compared with the acquisition of projective left/right in European children which is often not fully mastered till age 11 (Piaget and Inhelder, 1956). This result is consonant with other work on child spatial language showing that children's language use reveals very early sensitivity to language-specific semantic differences, adapting to the local system of spatial categories from the beginning (e.g., Bowerman, 1996; Bowerman and Choi, 2001). This is evidence that in this domain the child must construct the relevant categories; these are not given by innate endowment, contrary to what had been argued for the spatial domain. 


\section{How Spatial Distinctions Are Coded in Language Forms}

Much of the literature gives the impression that spatial distinctions are primarily coded in adpositions, as in the prepositions of English. In fact, in most languages spatial information is distributed throughout the clause. Some languages have a score or more local cases (nominal suffixes) covering roughly the same distinctions as English prepositions (e.g., Finnish or the NE Caucasian language Avar). Other languages use a combination of case and adposition (as in Tamil), or case and spatial nominal (as in many Australian languages). Special classes of spatial nominals (e.g., 'front,' 'back,' and 'side') do a lot of the work in isolating languages like Chinese, and they play special roles in other languages ( $\mathrm{cf}$ front in the English complex preposition in front of, and north in the adverbial use He went north). Many spatial oppositions are made constructionally, as when German prepositions like auf take the dative to indicate location but the accusative to indicate motion toward a goal.

Finally, verbs play a crucial role in spatial description. In the case of motion, there is often a special form class of motion verbs, and languages tend to conflate motion with manner (as in English crawl) or with direction or 'path' (as in Spanish salir: 'to go out'), but not generally both (Talmy, 1983, 2000). In many languages (e.g., Dutch), there is a small set of contrasting verbs (often derived from posture verbs) used to describe static location, encoding properties of the figure or ground, or both. Some languages have a larger set (as in Tzeltal), and in this case most of the semantic load is taken by the verbs, which may encode such specific details as 'be located in a bowl-shaped container' (Brown, 1994). Where the verbal component of locative statements is semantically specific in this way, it can impose a spatial categorization on objects in the world (e.g., long.thin.vertically.upright versus horizontally.extended.in.three.dimensions) that is depicted in gestures used when talking of putting and taking things; this provides a window on the possible cognitive effects of these categorizations (Ameka and Levinson, 2007). Even verbs without such spatial specificity - e.g., verbs of motion like 'go,' 'come,' 'enter,' and 'exit' (Wilkins and Hill, 1995; Kita, 1999), and of caused motion or placement verbs like 'put' and 'take' (Kopecka and Narasimhan, 2011; Slobin et al., 2011) display considerable cross-linguistic variability in their semantics. And even closely related languages may differ in their 'basic locative construction,' for example, in whether a preposition or a verb provides the unmarked construction for describing locative relations (as in Mayan languages; see Bohnemeyer and Brown, 2007).

In conclusion, it is clear that spatial concepts form a complex semantic field made up of structured linguistic subdomains. The semantic parameters involved may ultimately have a universal base, but the way in which the semantic subdomains are organized displays considerable cross-linguistic variation. There is also cross-linguistic variation in the way in which such distinctions are encoded in the form classes of languages.

There is now a great deal more information about spatial semantics in different languages than was available 30 years ago. This includes detailed descriptions of the language of space in different languages (e.g., de León and Levinson, 1992; Svorou, 1993; Dirven and Pütz, 1996; Danziger, 1998; Levinson and Wilkins, 2006), and areal surveys of spatial language (e.g., Levinson and Haviland, 1994 for Mayan languages; Senft, 1997 for Oceanic languages; and O'Meara and Pérez Báez, 2011 for Mesoamerican languages), as well as a number of cross-linguistic comparisons of spatial language in particular semantic domains, for example, landscape (Burenhult and Levinson, 2008), body parts (Majid et al., 2006), locative verbs (Ameka and Levinson, 2007), placement verbs (Kopecka and Narasimhan, 2011), and space/time metaphors (e.g., Majid et al., 2012). There is therefore much more visibility for the range of cross-linguistic variation that exists in the spatial domain and in the extension of spatial meanings to more abstract domains like time, kinship, and social relations. In addition, we now have some initial evidence for distinct patterns of neural activation in the brain for different frames of reference (Janzen et al., 2012).

This linguistic variation is especially interesting given the centrality of spatial concepts in human thinking - and indeed it has been shown that linguistic differences in frames of reference can make a difference to how humans memorize and reason nonlinguistically about space (Levinson, 1996, 1997, 2003; Pederson et al., 1998; Majid et al., 2004; Haun et al., 2011). There is robust evidence for several unrelated languages and cultures (ranging from Mexico to Australia to Africa to Nepal and Bali) that peoples' frame of reference usage in nonlinguistic cognition matches that of their language use. This linguistic variation and the associated interactional and cultural properties pose questions that are central to our understanding of how language and cognition are interrelated, how they are embedded in cultural practices, and how children's cognitive development may be shaped while they are learning a language.

See also: Human Spatial Orientation, Neural Basis of; Spatial Cognition During Infancy and Early Childhood Across Cultures, Development of; Spatial Cognition; Spatial Navigation; Virtual Reality and Spatial Cognition.

\section{Bibliography}

Ameka, F.K., Levinson, S.C. (Eds.), 2007. The typology and semantics of locative predication: posturals, positionals and other beasts. Linguistics 45 (5) (special issue).

Bloom, P., Peterson, M., Nadel, L., Garrett, M. (Eds.), 1996. Language and Space. MIT Press, Cambridge, MA.

Bohnemeyer, J., Brown, P., 2007. Standing divided: dispositional predicates and locative predications in two Mayan languages. Linguistics 45 (5-6), 1105-1151.

Bowerman, M., 1996. The origin of children's spatial semantic categories: cognitive vs. linguistic determinants. In: Gumperz, J.J., Levinson, S.C. (Eds.), Rethinking Linguistic Relativity. Cambridge University Press, Cambridge, UK, pp. 145-176.

Bowerman, M., Choi, S., 2001. Shaping meanings for language: universal and languagespecific in the acquisition of spatial semantic categories. In: Bowerman, M., Levinson, S.C. (Eds.), Language Acquisition and Conceptual Development. Cambridge University Press, Cambridge, pp. 475-511.

Brown, P., 1994. The INs and ONs of Tzeltal locative expressions: the semantics of static descriptions of location. Linguistics 32, 743-790.

Brown, P., 2001. Learning to talk about motion UP and DOWN in Tzeltal: is there a language-specific bias for verb learning? In: Bowerman, M., Levinson, S.C. (Eds.), Language Acquisition and Conceptual Development. Cambridge University Press, Cambridge, pp. 512-543. 
Brown, P., Levinson, S.C., 2000. Frames of spatial reference and their acquisition; in Tenejapan Tzeltal. In: Nucci, L., Saxe, G., Turiel, E. (Eds.), Culture, Thought, and Development. Erlbaum, Mahwah, NJ, pp. 167-197.

Brown, P., Levinson, S.C., 2009. Language as mind tools: learning how to think through speaking. In: Guo, J., Lieven, E.V., Budwig, N., Ervin-Tripp, S., Nakamura, K. Ozcaliskan, S. (Eds.), Crosslinguistic Approaches to the Psychology of Language: Research in the Tradition of Dan Isaac Slobin. Psychology Press, New York pp. 451-464.

Burenhult, N., Levinson, S.C. (Eds.), 2008. Language and landscape: geographic ontology in cross-linguistic perspective. Language Sciences 30 (2/3) (special issue).

Cablitz, G.H., 2002. The acquisition of an absolute system: learning to talk about space in Marquesan (Oceanic, French Polynesia). In: Clark, E.V. (Ed.), Papers of the 2002 Stanford Child Language Research Forum. CLSI, Stanford, CA, pp. 40-49.

Danziger, E. (Ed.), 1998. Language, Space, and Culture, Special Issue of Ethos, vol. 26 (1).

Dasen, P.R., Mishra, R.C., 2010. Development of Geocentric Spatial Language and Cognition: An Eco-Cultural Perspective. Cambridge University Press Cambridge, UK

Diven, R., Pütz, M. (Eds.), 1996. The Construal of Space in Language and Thought. De Gruyter, Berlin.

Haun, D.B.M., Rapold, C.J., Janzen, G., Levinson, S.C., 2011. Plasticity of human spatial memory: spatial language and cognition covary across cultures. Cognition 119, 70-80.

Haviland, J.B., 2000. Pointing, gesture spaces, and mental maps. In: McNeill, D. (Ed.), Language and Gesture. Cambridge University Press, Cambridge, pp. $13-46$

Haviland, J.B., 2003. How to point in Zinacantán. In: Kita, S. (Ed.), Pointing: Where Language, Culture, and Cognition Meet. Lawrence Erlbaum Associates, Mahwah, N.J./London, pp. 139-170.

Herskovits, A., 1986. Language and Spatial Cognition. Cambridge University Press, Cambridge, UK

Hunn, E., 1993. Columbia Plateau Indian place names: what can they teach us? Journa of Linguistic Anthropology 6 (1), 3-26.

Jammer, M., 1954. Concepts of Space: The History of Theories of Space in Physics. Harvard University Press, Cambridge, MA.

Janzen, G., Haun, D.B.M., Levinson, S.C., 2012. Tracking down abstract linguistic meaning: neural correlates of spatial frame of reference ambiguities in language. PLOS One 7 (2), e30657.

Kita, S., 1999. Japanese enter/exit verbs without motion semantics. Studies in Language 23 (2), 307-330.

Kita, S., Danziger, E., Stolz, C., 2001. Cultural specificity of spatial schemas, as manifested in spontaneous gestures. In: Gattis, M. (Ed.), Spatial Schemas and Abstract Thought. MIT Press, Cambridge, MA, pp. 115-146.

Kopecka, A., Narasimhan, B. (Eds.), 2011. Events of Putting and Taking: A Crosslinguistic Perspective. Benjamins, Amsterdam.

Landau, B., Jackendoff, R., 1993. 'What' and 'where' in spatial language and spatial cognition. Behavioural and Brain Sciences 16 (2), 217-265.

Le Guen, Olivier, 2011. Modes of pointing to existing spaces and the use of frames of reference. Gesture 11 (3), 271-307.

Levinson, S.C., 1996. Frames of reference and Molyneux's question: crosslinguistic evidence. In: Bloom, P., Peterson, M., Nadel, L., Garrett, M. (Eds.), Language and Space. MIT Press, Cambridge, MA, pp. 109-170.

Levinson, S.C., 1997. Language and cognition: the cognitive consequences of spatial description in Guugu Yimithirr. Journal of Linguistic Anthropology 7 (1), 98-131.
Levinson, S.C., 2003. Space in Language and Cognition: Explorations in Linguistic Diversity. Cambridge University Press, Cambridge, UK

Levinson, S.C., Haviland, J.B. (Eds.), 1994. Space in Mayan languages. Linguistics 32 (4/5) (special issue).

Levinson, S.C., Meira, S., 2003. Natural concepts' in the spatial topological domain adpositional meanings in crosslinguistic perspective: an exercise in semantic typology. Language 79 (3), 485-516.

Levinson, S.C., Wilkins, D. (Eds.), 2006. Grammars of Space: Explorations in Cognitive Diversity. Cambridge University Press, Cambridge, UK.

Lyons, J., 1977. Semantics. Cambridge University Press, Cambridge, UK.

de León, L., 1994. Exploration in the acquisition of geocentric location by Tzotzil; children (Special Issue). In: Haviland, J., Levinson, S.C. (Eds.), Spatial Conceptualization in; Mayan Languages, Linguistics, vol. 32(4/5), pp. 857-884.

de León, L., Levinson, S.C. (Eds.), 1992. Spatial description in Mesoamerican languages. Special Edition of Zeitschrift für Phonetik, Sprachwissenschaft und Kommunikationsforschung 45 (6).

Majid, A., Boroditsky, L., Gaby, A. (Eds.), 2012. Time in Terms of Space (Research Topic) (Special Issue). Frontiers in Cultural Psychology.

Majid, A., Bowerman, M., Kita, S., Haun, D., Levinson, S.C., 2004. Can language restructure cognition? The case for space. Trends in Cognitive Sciences 8 (3), 108-114.

Majid, A., Enfield, N.J., van Staden, M. (Eds.), 2006. Parts of the body: cross-linguistic categorization. Language Sciences 28 (2/3) (special issue).

Miller, G., Johnson-Laird, P., 1976. Language and Perception. Cambridge University Press, Cambridge, UK.

O'Meara, C., Pérez Báez, G., 2011. Spatial frames of reference in Mesoamerican languages. Language Sciences 33 (6), 837-852.

Pederson, E., Danziger, E., Wilkins, D., Levinson, S.C., Kita, S., Senft, G., 1998. Semantic typology and spatial conceptualization. Language 74, 557-589.

Piaget, J., Inhelder, B., 1956. The Child's Conception of Space. Routledge, London.

Senft, G. (Ed.), 1997. Referring to Space: Studies in Austronesian and Papuan Languages. Clarendon Press, Oxford.

Slobin, D.I., Bowerman, M., Brown, P., Eisenbeiss, S., Narasimhan, B., 2011. Putting things in places: developmental consequences of linguistic typology. In Bohnemeyer, J., J.Pederson, E. (Eds.), Event Representation in Language and Cognition. Cambridge University Press, Cambridge, pp. 134-165.

Svorou, S., 1993. The Grammar of Space. Benjamins, Amsterdam.

Talmy, L., 1983. How language structures space. In: Pick, H., Acredolo, L. (Eds.), Spatial Orientation: Theory, Research and Application. Plenum Press, New York.

Wilkins, D., Hill, D., 1995. When GO means COME: questioning the basicness of basic motion verbs. Cognitive Linguistics 6 (2), 209-259.

Talmy, L., 2000. Toward a Cognitive Semantics, vols. 1, 2. MIT Press, Cambridge, MA

\section{Relevant Websites}

http://www.acsu.buffalo.edu/ jb77/Mesospace.htm - Spatial language and cognition in Mesoamerica.

http://www.acsu.buffalo.edu/ jb77/Mesospace1b.html - Spatial language and cognition beyond Mesoamerica.

http://www.mpi.nl/news/news-archive/language-seems-crucial-to-think-about-space-1 Max Planck Institute for Psycholinguistics. 